\title{
Aortic Valve Papillary Fibroelastoma: A Sea Anemone in the Heart, A Case Report
}

\author{
Mohamed E. Taha, b, c, Janani Kumaresanª, b
}

\begin{abstract}
Cardiac papillary fibroelastomas are benign primary cardiac tumors usually arising from the normal components of the endocardium. Grossly they have a characteristic short pedicle and multiple papillary fronds similar to sea anemone. Clinically they have varying presentations ranging from asymptomatic to severe ischemic or embolic complications. We hereby present a case of a middle-aged man who presented with exertional dyspnea and palpitations and was discovered to have papillary fibroelastoma involving the aortic valve. The described case illustrates some of the presenting features, the workup and some of the management modalities that need to be implicated in cases of papillary fibroelastomas.
\end{abstract}

Keywords: Papillary fibroelastoma; Embolic complications; Echocardiogram; Anticoagulation

\section{Introduction}

Cardiac papillary fibroelastomas (CPFs) are benign primary cardiac tumors usually arising from the normal components of the endocardium. They are the most common valvular tumors of the heart [1]. Most of the cases are asymptomatic; however, it carries a high risk for embolic complications such as stroke and acute coronary syndromes [2]. Thereby, once detected, surgical resection is required to guard against future complications. We present a case of a middle-aged man who presented with exertional dyspnea and palpitations and was discovered to have papillary fibroelastoma involving the aortic valve. The patient was successfully treated with surgical excision and made an uneventful recovery and course upon follow-up.

Manuscript submitted June 18, 2019, accepted October 1, 2019

a Department of Internal Medicine, University of Nevada - Reno, School of Medicine, Reno, NV, USA

${ }^{b}$ These authors contributed equally to the work.

${ }^{\mathrm{c} C}$ Corresponding Author: Mohamed E. Taha, Department of Internal Medicine, University of Nevada - Reno, School of Medicine, Reno, NV, USA.

Email: M_tahir1985@yahoo.com

doi: https://doi.org/10.14740/cr818

\section{Case Report}

A 55-year-old Caucasian man with a history of tobacco abuse and bipolar disorder presented to our outpatient clinic with complaints of fluttering sensation in the middle of the chest aggravated on lying down and exertional dyspnea for few months duration. One year prior to his presentation, he had an unremarkable workup for ischemia and echocardiogram was performed for episodes of atypical chest pains. Physical examination was positive for bradycardia, nonetheless normal vital signs. Heart and lung examination was unremarkable during the visit.

As a part of the workup, he underwent EKGs, which were significant only for sinus bradycardia. A 24-h Holter monitoring for palpitations showed rare premature atrial and ventricular contractions without supraventricular tachycardia or ventricular tachycardia. Transthoracic echocardiogram (TTE) revealed a mass on the aortic valve without any left ventricular (LV) outflow obstruction. His LV function was normal. Transesophageal echocardiogram (TEE) delineated a wellpedunculated mass on the right coronary cusp as shown in Figure 1. Computed tomography angiography (CTA) revealed no significant coronary atherosclerosis (Fig. 2). Brain magnetic resonance imaging (MRI) was unremarkable.

He underwent open-heart surgery and complete tumor excision. The intraoperative finding revealed a pedunculated, mobile fibroelastoma attached to the free edge of the right coronary leaflet. The tumor was removed entirely intact and was sent for pathological examination. Grossly, the specimen was revealed to have a frond-like appearance. In gross pathology, it appeared as an ovoid, soft, somewhat gelatinous, irregularly shaped tan white fragment of tissue $(1.0 \times 0.5 \times 0.3 \mathrm{~cm})$, suggestive of a papillary fibroelastoma (Fig. 3). After surgery, the patient remained asymptomatic and a follow-up echocardiogram after surgery was normal.

\section{Discussion}

CPFs are the most common valvular tumors of the heart and the third common benign cardiac tumors after myxoma and lipoma [1]. They have a high propensity to affect the aortic valve, the LV outflow tract and the anterior mitral leaflet. It can present as a single or multiple lesions [3]. Histologically, CRFs are composed of an outer hyperplastic endothelial layer, an intermediate proteoglycans and a central avascular core, while grossly, they resemble a "sea anemone" (Fig. 4). They have a gelatinous membrane 


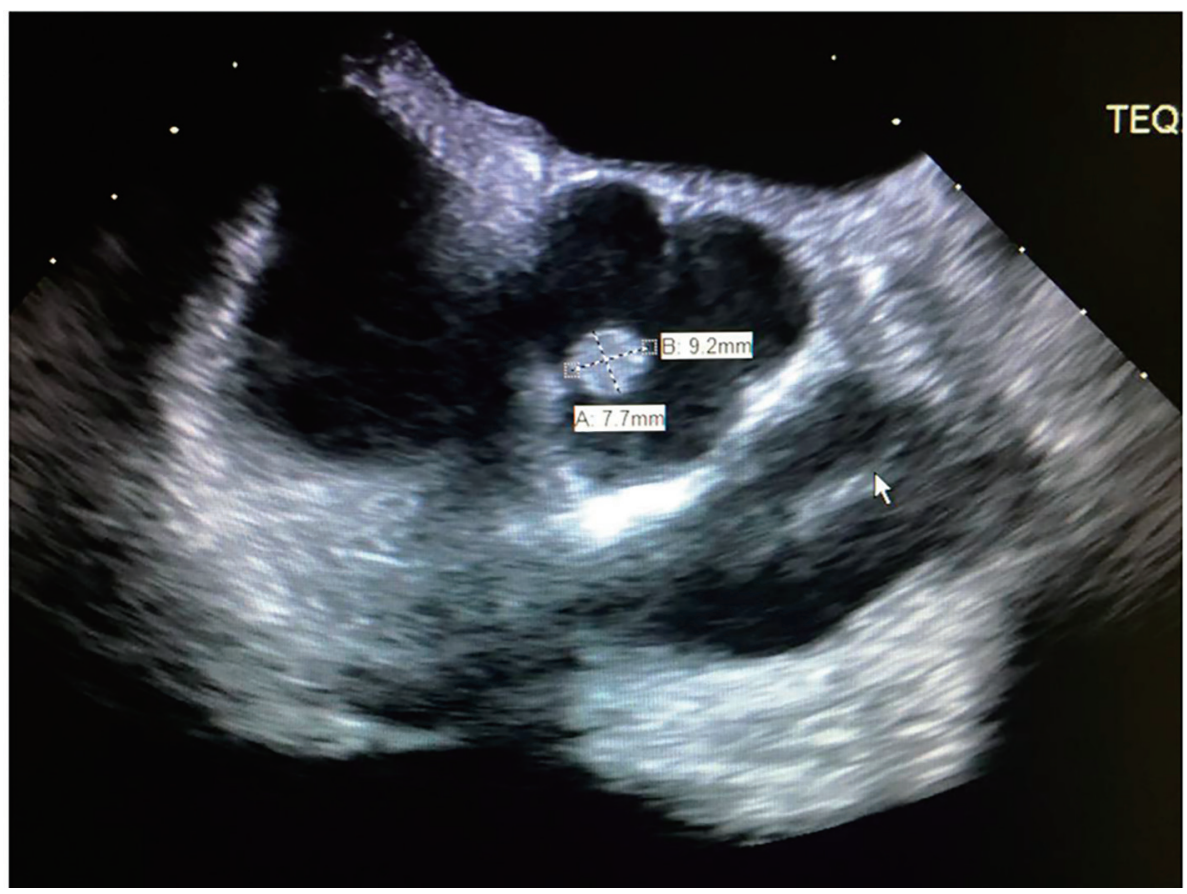

Figure 1. Transesophageal echocardiogram showing a pedunculated mass arising from the aortic valve.

on the surface and a stalk with multiple delicate papillary projections, best appreciated by immersing the specimen in water [4].

Clinically, most of CPFs are asymptomatic and often discovered incidentally in cardiac imaging. If needed symptoms are present, they usually result from central or peripheral embolization or through affecting the surrounding structures. The typical clinical manifestation is neurologic or cardiac, which includes transient ischemic attack, stroke, angina, myocardial infarction, sudden death, heart failure, syncope and blindness [2].

Diagnosis was usually accomplished through echocardiography, preferably TEE, which is safe and widely used currently as a method of diagnosing cardiac tumors overall [5]. Cardiac magnetic resonance (CMR) imaging can be used to enhance imaging when TEE is inconclusive. Coronary angi-

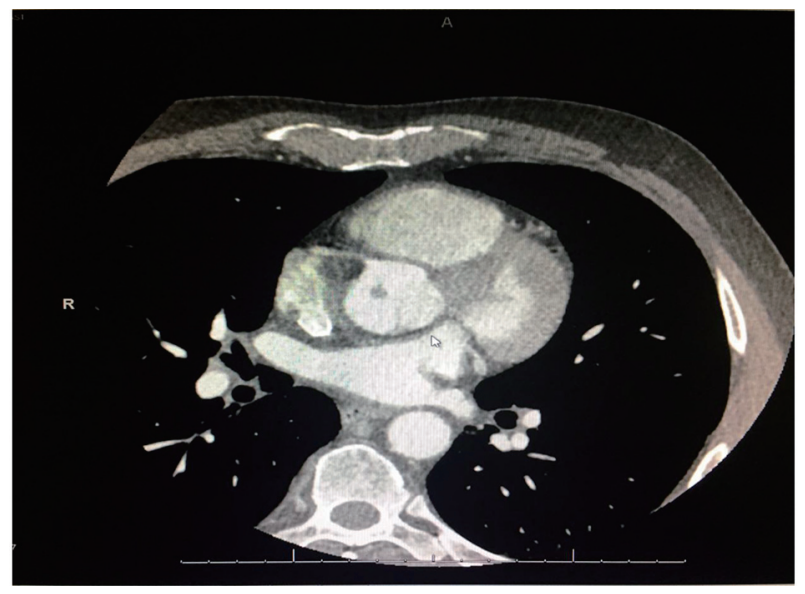

Figure 2. Computed tomography angiography showing no significant coronary atherosclerosis. ography or CT coronary angiography is used sometimes for diagnosis especially in patients over 40 years of age to rule out concomitant coronary artery disease.

There are no guidelines on the management of CPFs. However, there is general agreement on treating symptomatic patients with surgery. If surgical excision is delayed, anticoagulation therapy may be considered [6]. Surgical excision should be recommended for not only symptomatic patients but also for asymptomatic patients with high risk CPF to guard against serious and lethal complications. In this case, patient had a freely mobile papillary fibroelastoma with high susceptibility to embolization [7]. This case shows that papillary fibroelastomas can grow rapidly, with development of a mass within 2 years as seen on his repeat echocardiograms, and can progress to symptoms including palpitations. Our patient underwent surgical excision with removal of fibroelastoma, after which he made a good recovery with resolution of his symptoms.

\section{Conclusion}

Although papillary fibroelastomas are benign, it carries a high risk for devastating complications such as stroke, acute myocardial infarction, mesenteric ischemia and sudden death. Once papillary fibroelastoma is suspected, we recommend prompt echocardiography and anticoagulation to guard against surface thrombi, and early surgical referral.

\section{Acknowledgments}

We thank Dr. Rajindra Sarin, MD FACC from the Department 

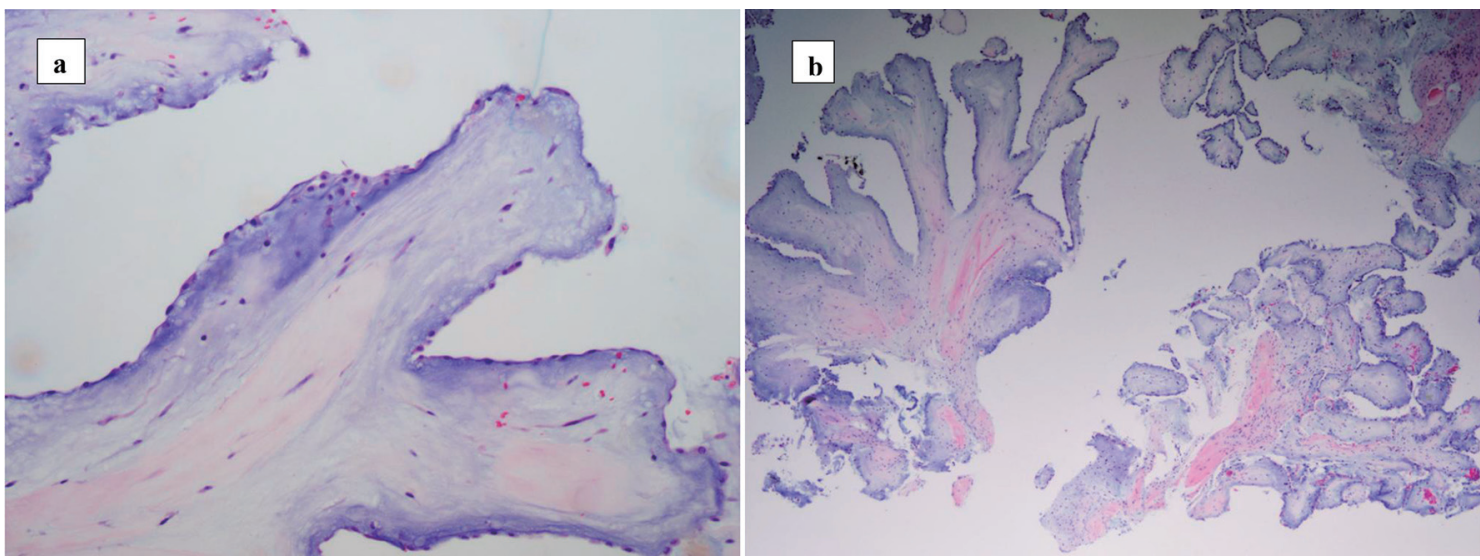

Figure 3. Histopathological specimen of the resected mass $(a, b)$ with characteristic features of papillary fibroelastoma.
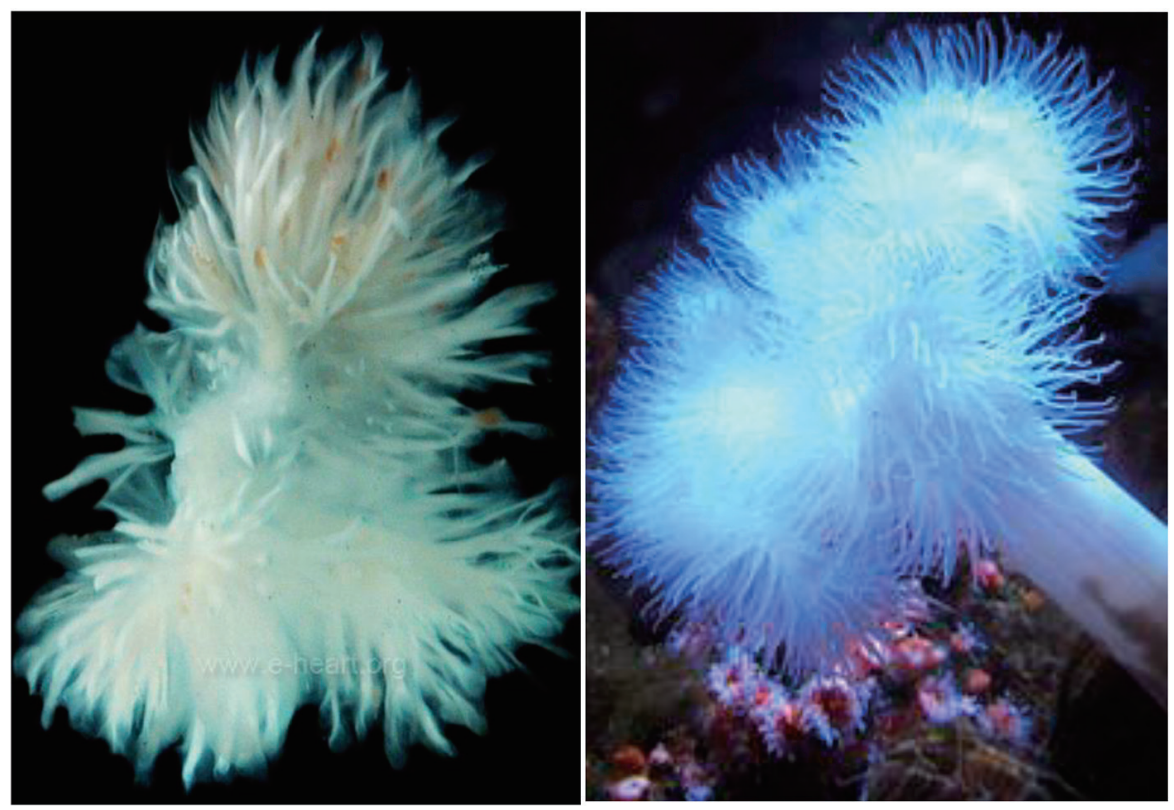

Figure 4. Gross specimen of papillary fibroelastoma (left) resembling a sea anemone (right).

of Cardiology in their expertise in taking care of the patient.

\section{Financial Disclosure}

The authors have not received any funding for this publication.

\section{Conflict of Interest}

All the authors declare that they have no conflict of interest.

\section{Informed Consent}

Informed consent to participate in the study was obtained from the patient. Consent was also obtained from the patient for the publication of materials related to this study.

\section{Author Contributions}

Mohamed Taha performed research including data collection and writing the paper. Janani Kumaresan performed research including data collection and provided expert clinical knowledge to revise critically.

\section{References}

1. Howard RA, Aldea GS, Shapira OM, Kasznica JM, Davidoff R. Papillary fibroelastoma: increasing recognition of a surgical disease. Ann Thorac Surg. 1999;68(5):1881-1885.

2. Ngaage DL, Mullany CJ, Daly RC, Dearani JA, Edwards 
WD, Tazelaar HD, McGregor CG, et al. Surgical treatment of cardiac papillary fibroelastoma: a single center experience with eighty-eight patients. Ann Thorac Surg. 2005;80(5):1712-1718.

3. Davoli G, Bizzarri F, Enrico T, Carone E, Muzzi L, Frati G, Chiavarelli M. Double papillary fibroelastoma of the aortic valve. Tex Heart Inst J. 2004;31(4):448-449.

4. Burke A, Virmani R. Tumors of the heart and great vessels. In: Atlas of Tumor Pathology. Third Series, Washington, DC, Armed Forces Institute of Pathology. 1996; vol 16; p. 47-54.

5. Engberding R, Daniel WG, Erbel R, Kasper W, Lestuzzi
C, Curtius JM, Sutherland GR, et al. Diagnosis of heart tumours by transoesophageal echocardiography: a multicentre study in 154 patients. European Cooperative Study Group. Eur Heart J. 1993;14(9):1223-1228.

6. Sakata T, Nakaya M, Otsu M, Sunazawa T, Wakabayashi Y. Autologous pericardial patch repair for papillary fibroelastoma on an aortic valve leaflet. Tex Heart Inst J. 2017;44(2):144-146.

7. Gowda RM, Khan IA, Nair CK, Mehta NJ, Vasavada BC, Sacchi TJ. Cardiac papillary fibroelastoma: a comprehensive analysis of 725 cases. Am Heart J. 2003;146(3):404410 . 